\title{
Prevalence of Chlamydia pneumoniae in Acute Respiratory Tract Infection and Detection of Anti-Chlamydia pneumoniae-specific IgE in Japanese Children with Reactive Airway Disease
}

\author{
SHIGERU IKEZAWA \\ Department of Pediatrics and Child Health, Kurume University School of Medicine, \\ Kurume 830-0011, Japan
}

\begin{abstract}
Summary: We examined the prevalence of Chlamydia pneumoniae in acute respiratory tract infection and association of $C$. pneumoniae infection and reactive airway disease in Japanese children. Four hundred eleven children with acute respiratory tract infection were enrolled in this study, and $C$. pneumoniae was isolated from $58(14.1 \%)$ patients by culture. Evidence of infection with $C$. pneumoniae was detected in 58 children with pneumonia (34.5\%), bronchitis $(41.4 \%)$ and upper respiratory tract infection $(24.1 \%)$. Twenty-nine $(50.0 \%)$ out of 58 patients were younger than 5 years old and $18(31.0 \%)$ had wheezing at first visit. A logistic test for anti-C. pneumoniae-specific $\lg \mathrm{E}$ showed the deference in the fluorescence unit between the patients with $C$. pneumoniae infection with and without wheezing was statistically significant $(\mathrm{Po}=0.02748$, to $=2.31891)$. In conclusion, $C$. pneumoniae seems to be an important respiratory tract pathogen among young Japanese children, and our results support the association of $C$. pneumoniae infection and reactive airway disease.
\end{abstract}

Key words Chlamydia pneumoniae, children, acute respiratory tract infection, asthma, anti- $C$. pneumoniae-specific IgE

\section{INTRODUCTION}

Chlamydia pneumoniae, also known as TWAR, was first described as a respiratory tract pathogen in 1986 [1], and it is emerging as a frequent cause of community-acquired respiratory tract infections, such as pharyngitis, bronchitis and pneumonia [2]. In addition, $C$. pneumoniae has been recently implicated in atherogenesis. $C$. pneumoniae has been isolated from $15-20 \%$ in adults and children presenting with respiratory tract symptoms in the USA $[3,4]$, however there is limited information on the prevalence of $C$. pneumoniae infection in Japan.

In 1991, Hahn et al. [5] reported an association between serologic evidence of acute $C$. pneumoniae infection and adult-onset asthma and asthmatic bronchitis. Emre et al. [6] demonstrated an association between the presence of anti-C. pneumoniaespecific $\operatorname{IgE}$ and wheezing during infection in children with reactive airway disease. The incidence of specific $\operatorname{IgE}$ was 86 and $22 \%$ in patients and controls, respectively, as estimated by immunoblotting. However, previous studies were hampered by labor-intensive and time-consuming detection techniques.

The aim of this study was to assess the role of $C$. pneumoniae in acute respiratory tract infections of children in southwestern Japan, and to determine if $C$. pneumoniae infection triggers the production of anti-C. pneumoniae-specific IgE utilizing ELISA, thus we examined sera from children with $C$. pneumoniae infection confirmed by culture. 


\section{PATIENTS AND METHODS}

\section{Patients and specimens}

Children between 1 month and 14 years of age with a diagnosis of acute respiratory tract infection, who were seen at Kurume University Hospital, Fukuoka, Japan, were prospectively examined. Kurume City is located in the southwestern part of Japan and has a population of approximately 230,000. Diagnosis of acute respiratory tract infection was based on clinical signs, symptoms and radiographic findings. Nasopharyngeal specimens were obtained from all patients using plastic-shafted dacron-tipped swabs for isolation of $C$. pneumoniae. Specimens were immediately placed in $1 \mathrm{ml}$ of sucrose-phosphate-glutamine transport media at $4{ }^{\circ} \mathrm{C}$ for $1-4 \mathrm{hrs}$ and stored at $-70{ }^{\circ} \mathrm{C}$. Acute phase sera were obtained from 21 patients for detection of antiC. pneumoniae-specific IgE and total IgE by ELISA.

\section{Culture}

Isolation of $C$. pneumoniae was performed by cycloheximide-treated HEp-2 cells grown in 96-well microtiter plates as previously described [7]. All specimens were passed once, and isolates were identified as chlamydiae with fluorescein-conjugated antibody to the lipopolysaccharide genus antigen (Pathfinder Chlamydia Culture Confirmation System; Kallestad Diagnostic, Chaska, Minn), and confirmed using $C$. pneumoniae-specific monoclonal antibody (Washington Research Foundation, Seattle, WA). Isolates were also stained by anti-major outer membrane protein (MOMP), Chlamydia trachomatisspecific monoclonal antisera (Syva, Palo Alto, CA) to identify C. trachomatis.

\section{Absorption of IgG}

Eighty-one microliters of test serum and $0.4 \mathrm{~mL}$ of a $25 \%(\mathrm{v} / \mathrm{v})$ suspension of recombinant protein Gagarose (Zymed Laboratories, San Francisco, CA) were mixed and shaken for one hour at room temperature, after centrifugation for $5 \mathrm{~min}$ at $2,000 \mathrm{~g}$, the supernatant (1:5 diluted serum) was subjected to the indirect ELISA for antibody activity.

Indirect ELISA for anti-Chlamydia pneumoniaespecific IgE

One hundred microliters of $10 \mu \mathrm{g} / \mathrm{mL}$ of purified TW-183 strain of $C$. pneumoniae (Washington Research Foundation, Seattle, WA) in coating buffer (0.05M Na-carbonate, $0.1 \% \quad \mathrm{NaN}_{3}, \mathrm{pH}$ 9.6) was added to the 96-well microtiter plate (MicroFLUOR: Dynex, Alexandria, UA) and incubated at $4{ }^{\circ} \mathrm{C}$ overnight. The plate was washed with PBS Tween (phosphate-buffer saline, 0.05\% Tween20, pH 7.4), and well was $0.1 \mathrm{~mL}$ of IgG-absorbed serum diluted 1:5 with BSA-PBS Tween $(1 \%(\mathrm{w} / \mathrm{v})$ bovine serum albumin) at room temperature for $3 \mathrm{hrs}$ while mixing. After washing, anti human IgE- $\beta$-galactosidase (CAP RAST FEIA kit, Pharmacia, Uppsala, Sweden) diluted with BSA-PBS Tween was added and plate was incubated at $4{ }^{\circ} \mathrm{C}$ overnight. After washing, 0.1 $\mathrm{mL}$ of $0.3 \mathrm{mM}$ 4-methylumbelliferyl- $\beta$-D-galactoside (Sigma Chemical Co., St.Louis, MO) in buffer A $\left(0.1 \mathrm{M} \mathrm{MgCl}_{2}, 0.1 \%\right.$ BSA, $10.1 \% \mathrm{NaN}_{3}, 0.01 \mathrm{M}$ Na-phosphate, $\mathrm{pH}$ 7.0) was added. The wells were sealed with tape, and the plates immersed in $37{ }^{\circ} \mathrm{C}$ water for $2 \mathrm{hrs}$. The enzyme reaction was stopped by adding $0.1 \mathrm{~mL}$ of $0.1 \mathrm{M}$ glycine- $\mathrm{NaOH}, \mathrm{pH} 10.2$. The fluorescence unit (FU) of each well was measured with a Fluorosakan II (Flow Laboratories, Rockuille, MD).

Tests for total $\operatorname{IgE}$ of serum were done with a VIDAS TOTAL IgE kit on mini VADAS (biome' rieux Vitek, MO) by enzyme linked fluorescent assay. The total IgE were calculated as prescribed by the manufacturer.

\section{Statistical analysis}

Statistical analysis for the comparison of serum anti- $C$. pneumoniae-specific IgE antibody was done using Aspin-Welch's t-test.

\section{RESULTS}

\section{Patients}

From January 1997 to December 1999, 411 children with acute respiratory tract infection were enrolled in this study. Their age ranged from 1 month to 14 years old and $196(47.7 \%)$ children were younger than 5 years old (Fig. 1). Evidence of infection with $C$. pneumoniae was detected in $58(14.1 \%)$ of the 411 patients by culture. Twenty $(34.5 \%)$ of these 58 culture-positive children had clinical and radiographic evidence of pneumonia, while 24 (41.4\%) children, who had evidence of lower respiratory tract infection on physical examination but did not have infiltrates on the chest X-ray film, were diagnosed as having bronchitis. In this study, clinical symptoms and findings of 58 patients with $C$. pneumoniae infection were as follows: 57 (98.3\%) of the patients had cough, 12 complained of moderate cough for $>3$ weeks and 18 had wheezing at first 


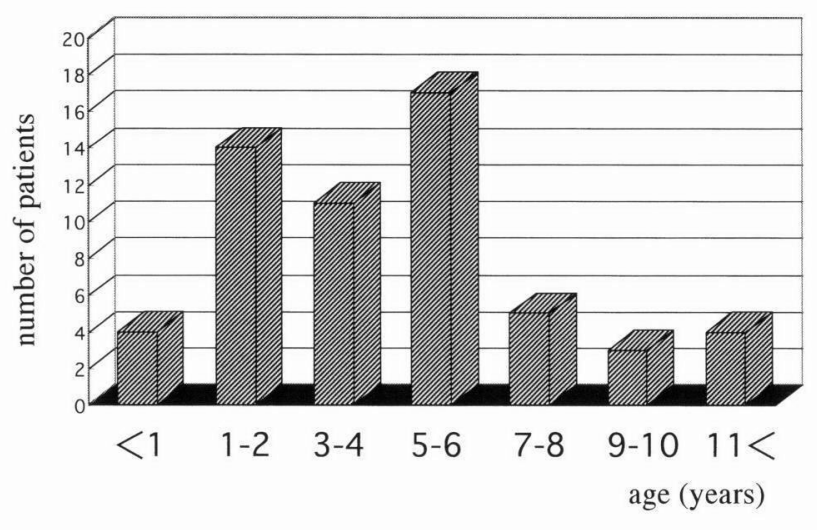

Fig. 1. Age distribution of 58 Chlamydia pneumoniae culture-positive patients with acute respiratory tract infection.

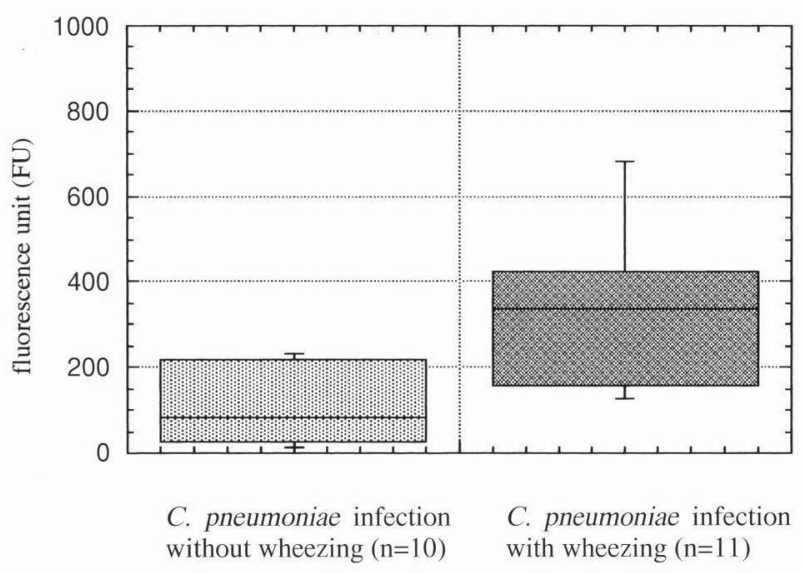

Fig. 2. Compare with fluorescence unit (FU) of anti-Chlamydia pneumoniae-specific IgE between the patients with $C$. pneumoniae infection with wheezing $(\mathrm{n}=11)$ and without wheezing $(\mathrm{n}=10)$. Data are shown as boxes: internal horizontal lines, medians; tops and bottoms of boxes, 25th and 75th percentiles, respectively. Upper and lower bars, 10th and 90th percentiles, respectively. $P$ values comparing the mean. (Aspin-Welch's t-test, $P_{O}=0.02748$, to $=2.31891$ )

visit; 29 (50.8\%) patients had fever of $>37.5^{\circ} \mathrm{C}$.

Indirect ELISA for anti-Chlamydia pneumoniaespecific $\operatorname{Ig} E$

We assessed 21 sera obtained from patients with $C$. pneumoniae infection with wheezing $(\mathrm{n}=11)$ and without wheezing $(\mathrm{n}=10)$. The sera of patients with wheezing elicited $267.50 \pm 36.85$ (mean $\pm S D$, range 127 to 682) fluorescence unit (FU), while that of patients without wheezing elicited 107.00 $\pm 90.70 \mathrm{FU}$ (range 15 to 232) (Fig. 2). The logistic AspinWelch's t-test showed the difference in FU between patients with and without wheezing was statistically significant. ( $\mathrm{Po}=0.02748, \mathrm{to}=2.31891$ ). There were not statistical differences between two groups in the total serum IgE (data not shown).

\section{DISCUSSION}

The prevalence of $C$. pneumoniae may vary according to geographic area, population density and age group examined. However, the information on the association between $C$. pneumoniae and respiratory tract infection in Japanese children is limited. A prior seroprevalence study done in Hiroshima, which involved 660 outpatients aged 7 months to 84 years, found that antibodies to $C$. pneumoniae increased rapidly between the age of 4 and 7 years. Forty-four percent of this study population had antibodies to $C$. pneumoniae by 8 to 11 years of age [8]. Numazaki et al. [9] studied 223 infants and children (aged 4 days to 14 years) with acute pneumonia utilizing MIF serology in Sapporo, in the north part of Japan. Eleven children $(4.9 \%)$ had $\operatorname{IgM}$ antibodies to $C$. pneumoniae. Ouchi et al. [10] examined 136 children (aged 2 months to 15 years) with acute lower respiratory tract infection living in Shimonoseki, in the southwestern part of Japan, also using MIF serology. Five $(3.7 \%)$ children met the serological criteria for acute $C$. pneumoniae infection. Polymerase chain reaction (PCR) was performed for throat swab specimens from these 5 children; 4 were positive.

In the present study, infection with $C$. pneumoniae was demonstrated in $58(14.1 \%)$ of 411 children with acute respiratory tract infection by culture. Our results demonstrated that $29(14.7 \%)$ out of 196 children under the age of 5 years had evidence of $C$. pneumoniae infection. C. pneumoniae seems to be an important respiratory tract pathogen among Japanese children in the Kurume area.

Previous studies have suggested that $C$. pneumoniae is a common lower respiratory tract pathogen among adults but less common among young children [11-17]. Most of these studies on the association between $C$. pneumoniae and acute lower respiratory tract infection were based on serology alone. In a study from Sudan [18], 3.6\% of 110 children with acute respiratory tract infection had either culture or serologic evidence of acute $C$. pneumoniae infection. In Germany [19], 1.0\% of 290 infants and children with acute respiratory tract infection were found to 
have acute $C$. pneumoniae infection utilizing culture and serological techniques and PCR. In contrast, the rates of $C$. pneumoniae infection in children with acute lower respiratory tract disease have been higher in several studies from the USA which utilized culture. In Brooklyn [3], 9 (12.2\%) of 74 patients less than 18 years old who presented with lower respiratory tract infection had $C$. pneumoniae infection by culture. In a nation-wide multicenter treatment study of community-acquired pneumonia, $42(16.2 \%)$ of 260 children were demonstrated to have $C$. pneumoniae infection based on culture [4]. This is probably reflective of improved isolation techniques. In the above mentioned multicenter study, infection with $C$. pneumoniae was detected in $28 \%$ of the 260 children by both culture and serology, however, only $23 \%$ of the culture positive children met the serologic criteria for acute $C$. pneumoniae infection [20]. Emre et al. [21] isolated $C$. pneumoniae from $13(11 \%)$ of 118 children with acute exacerbation of asthma and from $2(4.9 \%)$ of 41 asymptomatic controls. Seven $(58.3 \%)$ of 12 children with positive cultures also had no detectable antibody to $C$. pneumoniae and only $3(25 \%)$ of the culture positive children had serologic evidence of acute infection. The antibody response in acute $C$. pneumoniae infection may take longer than 3 weeks to develop [22]. Acute infection can also occur without seroconversion, especially in children [3,20,21]. Because of the relatively long period until the development of serologic response in primary infection, the microimmunofluorescence (MIF) test may not be sufficiently sensitive in children. The MIF test is generally accepted as the golden standard for the serological diagnosis of an acute $C$. pneumoniae infection. However, interpretation of specific and non-specific fluorescence patterns requires experience and skill, and the interpretation of high titer $(>512)$ is very difficult and subjective [23]. Moreover, cross-reactions between species of Chlamydia have been reported with the MIF assay [24-26]. Further prospective studies utilizing culture and PCR are required to clarify $C$. pneumoniae infection in children.

Infections with viruses, such as respiratory syncytial virus, parainfluenza virus and rhinovirus, have been identified in $40 \%-60 \%$ of children with acute exacerbation of asthma [6]. Although several viral and atypical pathogens have been linked with possible asthma initiation and promotion, the association of $C$. pneumoniae infection with asthma is the most extensively documented. Evidence of the role of $C$. pneumoniae in the pathogenesis of asthma has been obtained by culture isolation, PCR, serologically and by preliminary treatment trial. Most of the evidence for C. pneumoniae infection in adult-onset asthma has been based on serology, since successful culture isolation of $C$. pneumoniae from the upper airway of adults with stable chronic asthma has been only infrequently reported [27]. In our study, 18 (31\%) out of 58 children positive for $C$. pneumoniae by culture, had wheezing.

In 1996, Hahn et al. [27] reported that patients with symptomatic asthma had significantly more often IgA antibodies present in the serum than controls. Recently, Cook et al. [28] found that elevated IgG antibody levels to $C$. pneumoniae were significantly associated with severe chronic asthma, and this association was not modified by potentially confounding factors. In 1999, Hertzen et al. [29] reported the results of a logistic regression analysis that controlled for age, sex and smoking showing that asthma was significantly associated with elevated IgG antibody levels to $C$. pneumoniae (odd ratio 3.3). A number of mechanisms have been proposed to explain airway hyperresponsiveness following infection, including the development of organism-specific IgE antibody [6].

Emre et al. [21] investigated the prevalence of $C$. pneumoniae infection in children with acute exacerbation of reactive airway disease, and found that $C$. pneumoniae was isolated from $11 \%$ of children with acute episodes of wheezing compared with $4.9 \%$ of age- and sex-matched healthy controls. Subsequently, they demonstrated that anti-C. pneumoniae-specific IgE was associated with asthma in culture-positive children [6]. Specific IgE was detected in 86 and $22 \%$ of patients and controls, respectively, assessed by immunoblotting. In contrast to this study of children with asthma-like symptoms, Larsen et al. [30] examined adults diagnosed as having bronchial asthma and detected anti- $C$. pneumoniae-specific IgE in the patients as well as in the control group without differences in the pattern between the two groups. The prevalence of anti-C. pneumoniae-specific IgE was approximately $69 \%$ in both groups. In the present study, to further investigate the mechanism of $C$. pneumoniae-associated reactive airway disease, we examined sera from the children with $C$. pneumoniae infection with and without wheezing for the presence of anti-C. pneumoniae-specific IgE. Our results showed that anti-C. pneumoniae-specific IgE was detected significantly more frequently in the sera from the patients with wheezing than without wheez- 
ing, utilizing ELISA. Whereas, the total serum IgE level in patients with $C$. pneumoniae infection was not significant differences between the patients with wheezing and without wheezing. $C$. pneumoniae infection may be related to the development of chemical mediators leading to airway reactivity. Although the size of the population in this study was relatively small, our results support our clinical observations on the association of $C$. pneumoniae infection and reactive airway disease. The role of host factor as well as other immune mechanisms in the pathogenesis of $C$. pneumoniae infections needs to be further investigated.

ACKNOWLEDGMENTS: The author is grateful to Prof. Hirohisa Kato (Department of Pediatrics and Child Health, Kurume University School of Medicine) for giving the opportunity to perform this study and for his guidance and suggestions. He also thanks Dr. Naoki Tsumura (Department of Pediatrics and Child Health, Kurume University School of Medicine) for his advice regarding culture, Dr. Teruko Komoda, Dr. Hiroshi Miyazawa and Prof. Hisaichi Bannai (Department of Medical Technology, Kyorin University School of Health Science, Tokyo, Japan) for their advice regarding ELISA procedures and purified technique, Mr. Norikazu Kuriya (Department of Pediatrics and Child Health, Kurume University School of Medicine) for advice regarding analysis of data.

\section{REFERENCES}

1. Grayston JT, Kuo CC, Wang SP, and Altman J. A new Chlamydia psittaci strain, TWAR, isolated in acute respiratory tract infection. N Engl J Med 1986; 315:161168.

2. Grayston JT. Infections caused by Chlamydia pneumoniae strain TWAR. Clin Infect Dis 1992; 15:757-763.

3. Chirgwin K, Roblin PM, Gelling M, Hammerschlag MR, and Schachter J. Infection with Chlamydia pneumoniae in Brooklyn. J Infect Dis 1991; 163:757-761.

4. Roblin PM, Montalban G, and Hammerschlag MR. Susceptibility to clarithromycin and erythromycin of isolates of Chlamydia pneumoniae from children with pneumonia. Antimicrob Agents Chemother 1994; 38:1588-1589.

5. Hahn DL, Dodge RW, and Golubjatnikov R. Association of Chlamydia pneumoniae (Stain TWAR) infection with wheezing, asthmatic bronchitis, and adult-onset asthma. JAMA 1991; 266:225-230.

6. Emre U, Sokolovskaya N, Roblin PM, Schachter J, and Hammerschlag MR. Detection of Anti-Chlamydia pneumoniae IgE in children with reactive airway disease. J Infect Dis 1995; 172:265-267.

7. Roblin PM, Dumornay W, and Hammerschlag MR. Use of HEp-2 cells for improved isolation and passage of Chlamydia pneumoniae. J Clin Microbiol 1992; 30:19681971.
8. Kanamoto Y, Ouchi K, Mizui M, Ushio M, and Usui T. Prevalence of antibody to Chlamydia pneumoniae TWAR in Japan. J Clin Microbiol 1991; 29:816-818.

9. Numazaki K, Chiba S, and Umetsu M. Detection of IgM antibodies to Chlamydia trachomatis, Chlamydia pneumoniae, and Chlamydia psittaci from Japanese infants and children with pneumonia. In Vivo 1992; 6:601-604.

10. Ouchi K, Nakazawa T, Karita M, and Kanehara Y. Prevalence of Chlamydia pneumoniae in acute lower respiratory infection in pediatric population in Japan. Acta Paediatr Jpn 1994; 36:256-260.

11. Saikku P, Ruutu P, Leinonen M, Panelius J, Tupasi TE et al. Acute lower-respiratory-tract infection associated with chlamydial TWAR in Filipino children. J Infect Dis 1988; 158:1095-1097.

12. Grayston JT, Diwan VK, Cooney M, and Wang SP. Community- and hospital-acquired pneumonia associated with Chlamydia TWAR infection demonstrated serology. Arch Inter Med 1989; 149:169-173.

13. Wang SP, and Grayston JT. Population prevalence antibody to Chlamydia pneumoniae. In: Chlamydia Infections: Proceeding of the Seventh International Symposium on Human Chlamydia Infections, ed. Bowie et al., Cambridge University Press, Cambridge, England, pp 402-405, 1990.

14. Aldous MB, Grayston JT, Wang SP, and Foy HM. Seroepidemiology of Chlamydia pneumoniae TWAR infection in Seattle families, 1966-1979. J Infect Dis 1992; 166:646-649.

15. Carballal G, Mahony JB, Videla C, Cerqueiro C, and Chernesky M. Chlamydial antibodies in children with lower respiratory disease. Pediatr Infect Dis J 1992; 11:68-71.

16. Yeung SM, McLeod K, Wang SP, Grayston JT, and Wang EEL. Lack of evidence of Chlamydia pneumoniae infection in infants with acute lower respiratory tract disease. Eur J Clin Microbiol Infect Dis 1993; 12:850853 .

17. Grayston JT. Chlamydia pneumoniae (TWAR) infections in children. Pediatr Infect Dis J 1994; 13:675-685.

18. Herrmann B, Salih MAM, Yousif BE, Abdelwahab O, and Mårdh P-A. Chlamydial etiology of acute lower respiratory tract infections in children in the Sudan. Acta Paediatr 1994; 83:169-172.

19. Jantos CA, Wienpahl B, Schiefer HG, Wagner F, and Hegemann JH. Infection with Chlamydia pneumoniae in infants and children with acute lower respiratory tract disease. Pediatr Infect Dis J 1995; 14:117-122.

20. Block S, Hedrick J, Hammerschlag MR, Cassell GH, and Craft JC. Mycoplasma pneumoniae and Chlamydia pneumoniae in pediatric community-acquired pneumonia: comparative efficacy and safety of clarithromycin vs. erythromycin ethylsuccinate. Pediatr Infect Dis J $1995 ; 14: 471-477$.

21. Emre U, Roblin PM, Gelling M, Dumornay W, Hammerschlag MR et al. The association of Chlamydia pneumoniae infection and reactive airway disease in children. Arch Pediatr Adolesc Med 1994; 148:727-732.

22. Grayston JT, Campbell LA, Kuo CC, Mordhort $\mathrm{CH}$, Saikku $\mathrm{P}$ et al. A new respiratory pathogen: Chlamydia 
pneumoniae strain TWAR. J Infect Dis 1990; 161:618625.

23. Verkooyen RP, Van Lent NA, Mousavi Joulandan SA, Snijner RJ, Van Den Bosch JM et al. Diagnosis of Chlamydia pneumoniae infection in patients with chronic obstructive pulmonary disease by micro-immunofluorescence and ELISA. J Med Microbiol 1997; 46:959-964.

24. Kern DG, Neill MA, and Schacter J. A seroepidemiologic study of Chlamydia pneumoniae in Rhode Island. Evidence of serologic cross-reactivity. Chest 1993; 104:208-213.

25. Moss TR, Darougar S, Woodland RM, Nathan M, Dines DJ et al. Antibodies to chlamydia species in patients attending a genitourinary clinic and the impact of antibodies to Chlamydia pneumoniae and Chlamydia psittaci on the sensitivity and the specificity of Chlamydia trachomatis serology tests. Sex Trans Dis 1993; 20:61-65.

26. Hyman CL, Roblin PM, Gaydos MS, Quinn TC, Schachter $\mathbf{J}$ et al. The prevalence of asymptomatic nasopharyngeal carriage of Chlamydia pneumoniae in subjectively healthy adults: assessment by polymerase chain reaction-enzyme immunoassay and culture. Clin Infect Dis 1995; 20:1174-1178.

27. Hahn DL, Anttila T, and Saikku P. Association of Chlamydia pneumoniae IgA antibodies with recently symptomatic asthma. Epidemiol Infec 1996; 117:513517.

28. Cook PJ, Davies P, Tunnicliffe W, Ayres JG, Honeybourne D et al. Chlamydia pneumoniae and asthma. Thorax 1998; 53:254-259.

29. Von Hertzen L, To “yryla” M, Gimishanov A, Bloigu A, Leinonen $\mathrm{M}$ et al. Aathma, atopy and Chlamydia pneumoniae antibodies in adults. Clin Exp Allergy 1999; 29:522-528.

30. Larsen FO, Norn S, Mordhorst $\mathrm{CH}$, Stahl Skov P, Milman N et al. Chlamydia pneumoniae and possible relationship to asthma. APMIS 1998; 106:928-934. 